\title{
Reducing barriers to ethics in neuroscience
}

\author{
Judy IIles ${ }^{1 *}$, Kate Tairyan ${ }^{1}$, Carole A. Federico ${ }^{1}$, Aline Tabet ${ }^{2}$ and Gary H. Glover ${ }^{3}$ \\ 1 National Core for Neuroethics, Division of Neurology, Department of Medicine, The University of British Columbia, Vancouver, BC, Canada \\ 2 Department of Statistics, The University of British Columbia, Vancouver, BC, Canada \\ ${ }^{3}$ Radiological Sciences Lab, Department of Radiology, Stanford University School of Medicine, Stanford University, Stanford, CA, USA
}

\section{Edited by:}

Hauke R. Heekeren, Max Planck

Institute for Human Development,

Germany

\section{Reviewed by:}

Georg Northoff, University of Ottawa, Canada

Christian Hoppe, University of Bonn

Medical Centre, Germany

${ }^{*}$ Correspondence:

Judy Illes, National Core for

Neuroethics, University of British

Columbia, 2211 Wesbrook Mall,

Koerner S124, Vancouver, BC, Canada

V6T 2B5.

e-mail: jilles@interchange.ubc.ca
Ethics is a growing interest for neuroscientists, but rather than signifying a commitment to the protection of human subjects, care of animals, and public understanding to which the professional community is engaged in a fundamental way, interest has been consumed by administrative overhead and the mission creep of institutional ethics reviews. Faculty, trainees, and staff ( $n=605$ ) in North America whose work involves brain imaging and brain stimulation completed an online survey about ethics in their research. Using factor analysis and linear regression, we found significant effects for invasiveness of imaging technique, professional position, gender, and local presence of bioethics centers. We propose strategies for improving communication between the neuroscience community and ethics review boards, collaborations between neuroscientists and biomedical ethicists, and ethics training in graduate neuroscience programs to revitalize mutual goals and interests.

Keywords: neuroimaging, neuroethics, ethics

\section{INTRODUCTION}

Ethics is a growing interest for neuroscientists but is also battling a bad reputation that it cannot seem to shake. Rather than signifying a commitment to the protection of human subjects, care of animals, and public understanding to which the professional community is engaged in a fundamental way, interest in the moral underpinnings of research has been overtaken by administrative overhead and the mission creep of institutional ethics reviews beyond their original, intended goals (Gunsalus et al., 2006). Regulation and obligation have taken the breath out of researcher good will and compromised efforts to integrate fully societal concerns and potential implications of new research results upstream into the experimental process. This disconnect has important consequences for brain research, as advances in neuroscience are having a more direct impact on the understanding of and approach to brain health and brain disease than ever before (Hyman, 2004).

The obligation to take note of societal implications of neuroscience has been expressed in a growing number of publications (Caplan, 2002; Illes and Racine, 2005; Leshner, 2005; Illes, 2006; Illes and Bird, 2006; Garnett et al., 2010), in professional conferences and the explicit integration of lectures and symposia at the Society for Neuroscience (SfN), Federation of European Neuroscience Societies (FENS), the Organization of Human Brain Mapping (OHBM), and the American Academy of Neurology (AAN), and through the development of professional societies (e.g., Neuroethics Society www.http://neuroethicssociety.org, and the Society for Social Neuroscience www.http://s4sn.org) devoted to these issues.

Within the broad domain of brain research, imaging is an important focus for these discussions as new results have informed how people think, make decisions and hold beliefs, have brought new meaning to old diagnostic categories in neurology and mental health, have affected medical-legal decision-making, and opened new doors to personalized medicine both within traditional health care systems and in the open marketplace. With prospects for the powerful combination of neuroimaging with other modalities such as genetics and molecular pharmacology, there is an even greater need for rigorous exploration of the ethical dimensions of research at present and on the horizon (Tairyan and Illes, 2009; Buchman and Illes, 2010).

Here we sought to characterize the landscape of ethics in neuroimaging as one domain of neuroscience. Using North America as a starting point, we asked principal investigators (PIs) and faculty members whose work specifically involves brain imaging and stimulation, and their students and professional staff, what they view as key challenges related to ethics in their work, what motivates them to give consideration to those issues, and the barriers to do so beyond the requirements for institutional approval in research.

\section{MATERIALS AND METHODS}

We invited 1200 PIs based in the United States and Canada whose research involves electrophysiology (e.g., electroencephalography [EEG]), magnetic resonance imaging (MRI and functional MRI), magnetoencephalography (MEG), positron and single photon emission tomography (PET and SPECT), optical imaging (NIR), deep brain stimulation (DBS), transcranial magnetic stimulation (TMS), or imaging genetics to participate in an online survey. PIs were identified through searchable databases of federally funded research grants of the US-based National Institutes of Health (NIH, Computer Retrieval of Information on Scientific Projects [CRISP]), the Canadian Institute of Health Research (CIHR), the Natural Sciences and Engineering Research Council of Canada (NSERC), and the Social Sciences and Humanities Research Council of Canada (SSHRC). To capture responses from investigators with current research activity, we restricted the search to grants awarded between 2007 and 2009 inclusive. Researchers were asked to forward the invitation to participate on to others in their research team 
for their voluntary and anonymous participation. Approval for this study was obtained from the University of British Columbia Behavioral Research Ethics Board.

With an online survey containing 45 questions (please see Supplementary material), we probed investigators on the relative importance of ethics issues they encounter in their work, and what motivates and prevents them from incorporating ethics into their research. Answers were given based on a five-point Likert scale with opportunity for augmenting the quantitative responses with free-text, narrative content. We used exploratory factor analysis to transform the original questionnaire items into a smaller set of factors, reducing the dimensionality of the data and achieving meaningful interpretations about ethics constructs in neuroimaging. We applied factor analysis with a varimax rotation separately on the items addressing ethical issues, motivators and barriers. In each case, the number of factors was chosen based on the Kaiser rule of retaining all factors with a corresponding eigenvalue greater than 1. Sampling adequacy was assessed using the Kaiser-Meyer-Olkin (KMO) test that, for each of the three cases, was over 0.8. Bartlett's method was used to obtain unbiased estimates of the factor scores for each respondent computed as a weighted sum of the original variables. We used a linear regression model to understand the effect of key variables of interest on the factor scores.

\section{RESULTS}

\section{RESPONDENTS}

We received 605 responses to the survey from principal investigators and faculty, postdoctoral fellows, graduate students, and staff: $47 \%$ are female; $42 \%$ hold faculty positions, $61 \%$ have a $\mathrm{PhD}$ and/ or MD degree, and 35\% are the head of their research group. Of the cohort of responders, $81 \%$ report that their studies involve human subjects only, $5 \%$ involve non-human primates and other animals, and the remainder use two or more models. The majority of respondents use functional MRI (79\%) alone or in combination with other techniques.

\section{ETHICS-RELATED ISSUES}

Key ethics-related issues reported by the overall group cluster into four factors (Table 1): 1. confidentiality and consent (14.2\% of variance), 2. external forces on academic productivity (13.5\% of variance), 3. conflict of interest ( $11 \%$ of variance), and 4 . vulnerability and expectations ( $8 \%$ of variance). The first effect we note from the regression analysis with these factors (Table 2 ) is for respondents who expressed a high interest in ethics and a high satisfaction with available ethics resources: they view challenges involving confidentiality and consent as more important than respondents in the reference categories (no interest in ethics; no satisfaction with ethics resources; $t(520)=2.08, p<0.05 ; t(520)=2.57, p<0.05)$. Postdoctoral fellows view confidentiality and consent as a less important ethics challenge than faculty $(t(520)=-2.07, p<0.05)$; the inverse is the case for staff $(t(520)=2.36, p<0.05)$. Women researchers rate the importance of matters related to recruitment, confidentiality, and privacy of human subjects more highly than men $(t(520)=2.37, p<0.05)$ and are relatively less concerned with conflict of interest $(t(520)=-4.07, p<0.01)$. We observe a similar positive effect for women neuroimagers related to the recruitment of vulnerable populations, expectations of subjects, and incidental findings $(t 520)=3.21, p<0.01)$. For this effect, the finding is the inverse for both staff $(t(520)=-2.11, p<0.05)$ and students $(t(520)=-2.40, p<0.05)$ compared to faculty.

\section{MOTIVATORS}

Key motivators for considering ethics issues in neuroimaging cluster into five groups (Table 1): 1. external factors (16\% of variance), 2. trust and reciprocity (15\% of variance), 3. requirements $(12 \%$ of variance), 4 . virtue (10\% of variance), and 5 . communication ( $8 \%$ of variance). Faculty are more motivated to consider ethics issues by the factor we called virtue than postdoctoral fellows $(t(538)=2.74$, $p<0.01)$ and students $(t(538)=2.69, p<0.01)$. Female researchers value trust and reciprocity more than their male counterparts $(t(538)=4.48, p<0.01)$. Postdoctoral fellows $(t(538)=4.24, p<0.01)$, staff $(t(538)=5.22, p<0.01)$ and students $(t(538)=5.02, p<0.01)$ are motivated by external factors such as professional advancement, institutional encouragement, and publication success. Respondents who have access to a local bioethics center $(t(538)=2.00, p<0.05)$ and those who are satisfied with bioethics resources $(t(538)=2.75$, $p<0.01)$ are more motivated by the requirements of their institutions and research sponsors than those who do not. Requirements are also a significant factor motivating investigators whose research involves brain stimulation $(t(538)=2.65, p<0.01)$ compared to researchers who use non-invasive techniques.

\section{BARRIERS}

Barriers to incorporating ethics into neuroimaging research cluster into four factors: 1 . lack of resources (17\% of variance), 2. administrative burden (15\% or variance), 3 . indifference (15\% of variance), and 4 . lack of interest ( $13 \%$ of variance). Postdoctoral fellows $(t(546)=2.65$, $p<0.01)$, staff $(t(546)=3.41, p<0.01)$ and students $(t(546)=3.86$, $p<0.01)$ rated indifference as an important barrier compared to faculty. In addition, men rated indifference to be a more significant barrier than women $(t(546)=2.75, p<0.01)$. Administrative burden is a considerable barrier for those who use invasive methods in comparison to those who do not $(t(546)=2.32, p<0.05)$, and for those whose institutions house a bioethics center $(t(546)=2.79, p<0.01)$. Lack of ethics resources is a particular barrier for postdoctoral fellows $(t(546)=2.40$, $p<0.05)$ and staff $(t(546)=2.04, p<0.05)$ compared to faculty.

\section{DISCUSSION}

Despite the core values of faculty to be good global citizens of research, the overall administrative burden of ethics review seem to place them at the mercy of the boards to which oversight falls. As one respondent commented in the survey:

\begin{abstract}
"The Investigational [sic] Review Board is often a barrier to ethical research. [...] The time spent by the investigators responding to issues raised by the IRB unrelated to ethical issues takes time away from ensuring the safety of subjects and often delays the implementation of important research" (Respondent \#103).
\end{abstract}

\section{Another respondent wrote:}

\footnotetext{
"[...] ethics regulations become kind of a running joke since they are so complex, take so long to get through, involve so much paperwork and regulation, and are sometime[s] almost impossible to follow to the level they impose [...]" (Respondent \#218).
} 
Table 1 | Exploratory factor analysis of ethics-related issues, motivators and barriers.

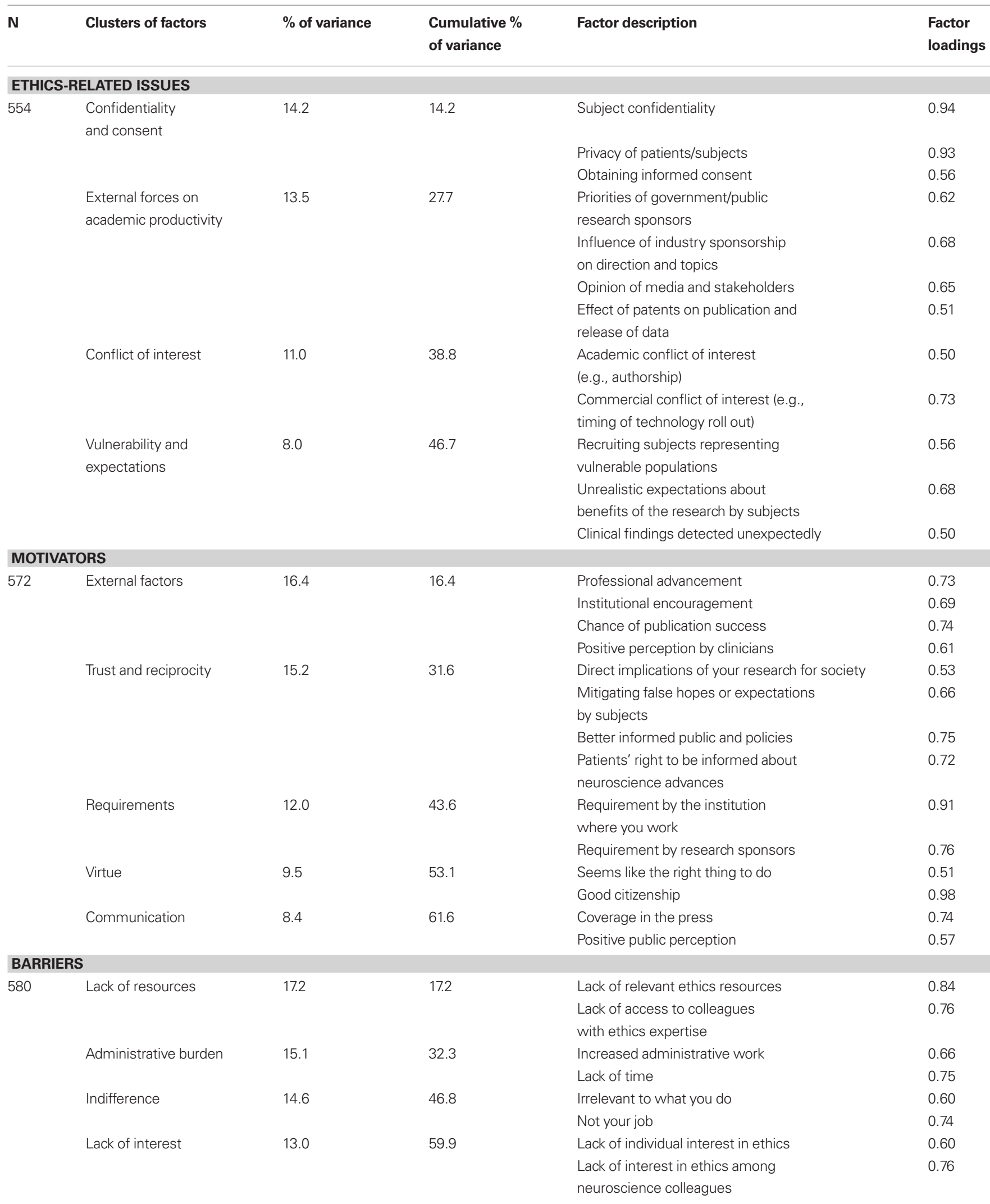

Factor analysis is based on complete data for each category; surveys with missing observations were excluded. The three factor analyses (issues, motivators, barriers) were fit separately. 


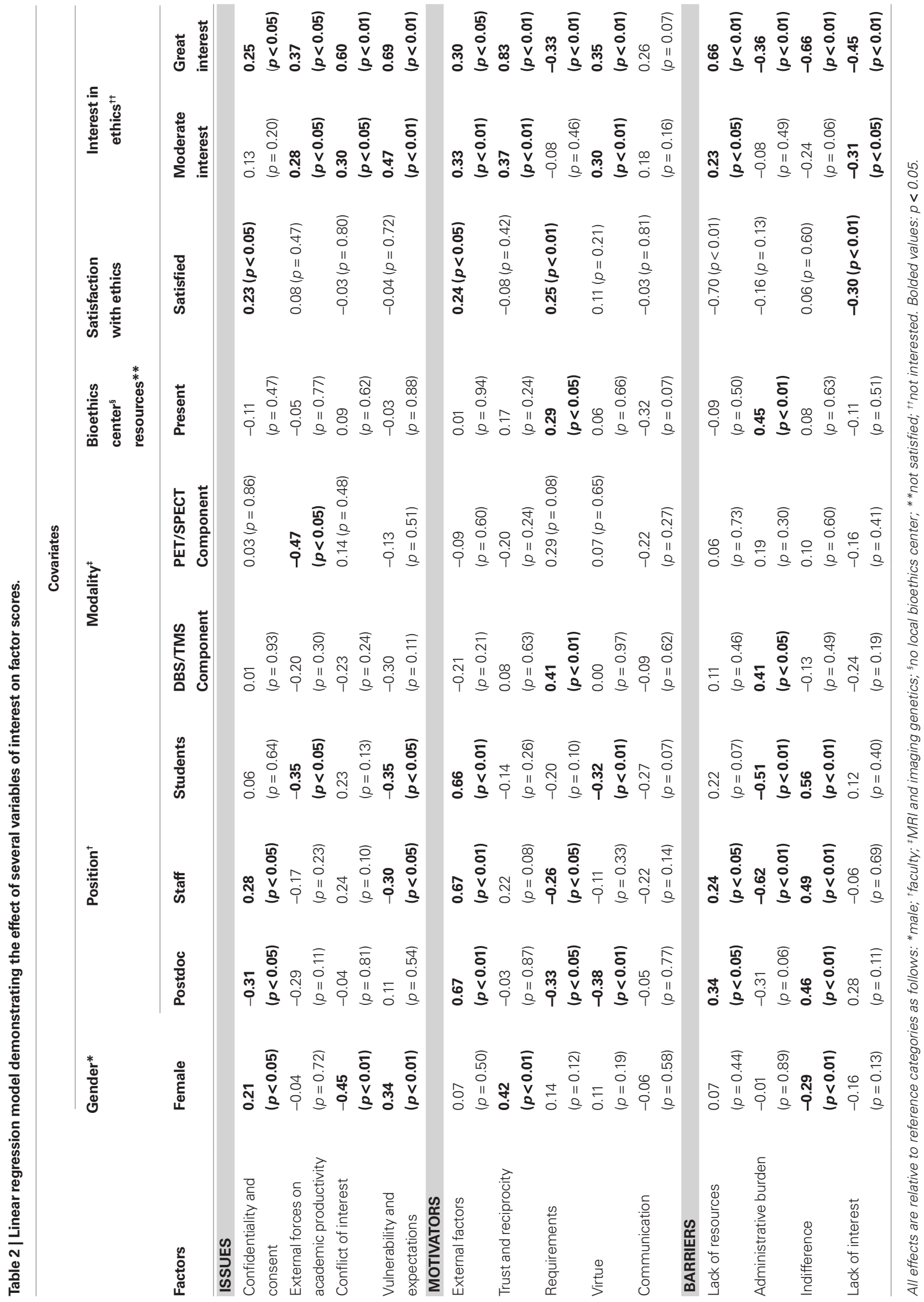


Focus group data from a parallel study mirror such sentiments; labeled as "unnecessary", "complex" and overregulated", the ethics review process is reported to be taking valuable resources away from researchers (Tairyan et al., 2010). Deslauriers et al. (2010) also reported a range of practical issues with the ethics review process, including the lack of guidance to investigators on the writing of informed consent documents, lack of expertise on ethics review boards to evaluate neuroimaging protocols, variability between and within review boards, and time delays in ethics review. Data from the present study suggest that trainees are relatively unaffected by this administrative burden and are the most indifferent to ethics issues overall. To the extent that they are motivated to think about ethics content, that motivation is closely tied to career advancement. A lack of resources is a key barrier to engaging this cohort more deeply. Local centers devoted to scholarly inquiry in biomedical ethics have no measurable effect on the issues, motivators or barriers in neuroimaging and, in fact, may be confused with institutional boards for ethics review. The source of the gender effect will require further study, but may be associated with the leadership and organizational skills of women that have been characterized as facilitating positive group dynamics and mutual respect (Lewis, 2000; Zielinska, 2010).

We recognize the limitations of this study, including the $18 \%$ response rate of PIs and the non-randomness of the sample; these limitations naturally restrict the generalizability of the results to the broader context of neuroscience and even science at large. However, in a parallel study of another sector of the neuroscience community - investigators whose primary research is focused on neurodegenerative diseases - we found similar effects to those we report here. Barriers from that study cluster into four familiar factors: administrative burden, lack of resources, indifference, and lack of interest. We found that the lack of ethics resources is a particular barrier for senior level investigators in this cohort and for researchers who work in the area of drug discovery (Federico et al., 2010). Although these were also only North American investigators, the data suggest an emerging pattern across neuroscience subspecialty categories. We also do not deliver a measure of the degree to which the barriers reported are burdensome. Continued empirical work

\section{REFERENCES}

Buchman, D.Z., and Illes, J. (2010). Imaging genetics for our neurogenetic future. Minn. J. Law, Sci. Technol. 11, 79-97.

Caplan, A. L. (2002). The brain revolution and ethics. The Scientist 16, 12.

Deslauriers, C., Bell, E., Palmour, N., Pike, B., Doyon, J., and Racine, E. (2010). Perspectives of Canadian researchers on ethics review of neuroimaging research. J. Empir. Res. Hum. Res. Ethics 5, 49-66.

Federico, C. A., Tairyan, K., Ivinson, A., and Illes, J. (2010). Untapped ethics resources for neurodegeneration research. Poster session to be presented at: Society for Neuroscience, November 13-17; San Diego, CA.

Garnett, A., Piwowar, H. A., Rasmussen, E. M., and Illes, J. (2010). Formulating MEDLINE queries for article retrieval based on PubMed exemplars. Nat. Proc. Available from: http://hdl.handle.net/10101/npre.2010.4270.2.
Gunsalus, C. K., Bruner, E. M., Burbules, N. C., Dash, L., Finkin, M., Goldberg, J. P., Greenough, W. T., Miller, G. A., and Pratt, M. G. (2006). Mission creep in the IRB world. Science 312, 1441.

Hyman, S. E. (2004). Introduction, the brain's special status. Cerebrum 6, 9-12.

Illes, J. (Ed.) (2006). Neuroethics: Defining the Issues in Theory, Practice and Policy. Oxford, UK: Oxford University Press.

Illes, J., and Bird, S. J. (2006). Neuroethics: a modern context for ethics in neuroscience. Trends Neurosci. 29, 511-517.

Illes, J., and Racine, E. (2005). Imaging or imagining? A neuroethics challenge informed by genetics. Am. J. Bioeth. 5, 5-18.

Leshner, A. I. (2005). It's time to go public with neuroethics. Am. J. Bioeth. 5, $1-2$. of this nature, including studies of larger and international cohorts, will clearly expand the knowledge base and solutions to the issues highlighted here.

These limitations notwithstanding, there are global strategies to overcome the challenges discovered here, and many of them will become pressing as neuroimaging increasingly touches the daily lives of people, informs social and public policy, and enables more effective and albeit potentially more invasive treatments of brain disorders. Among these strategies is the development and implementation of a new social contract (Samarasekera, 2009) between the neuroscience community and institutional review boards that moves these groups beyond the mistrust that has built up over time and that has unglued meaningful partnership. Ownership of the effort and implementation of solutions is a joint responsibility. Better communication between ethics committee members and researchers, and within ethics committees themselves about the mandate of reviewers will streamline ethics approval processes. The integration of relevant, culturally appropriate curricula early into graduate training will strengthen the ethics knowledge base of young researchers and embed the imperative for ethics in their research (Lombera et al., 2010). Collaborations between scholars in ethics and in neuroscience must be supported and valued.

Will the implementation of these solutions and others have a positive impact on research conduct, outreach to the public, and public trust? The upfront investment will be significant and outcomes will need to be measured empirically, but we predict that the answer is yes.

\section{ACKNOWLEDGMENTS}

Generously supported by NIH/NIMH R01 \#9R01MH84282, CIHR/CNE \#85117, Canadian Foundation for Innovation (CFI) and British Columbia Knowledge Development Fund (BCKDF). We thank Daniel Buchman and Emily Borgelt for comments on earlier versions of this manuscript.

\section{SUPPLEMENTARY MATERIAL}

The Supplementary Material for this article can be found online at http://www.frontiersin.org/neuroscience/humanneuroscience/ paper/10.3389/fnhum.2010.00167/

Lewis, K. M. (2000). When leaders display emotion: how followers respond to negative emotional expression of male and female leaders. J. Organ. Behav. 21, 221-234.

Lombera, S., Fine,A., Grunau, R.E., and Illes, J. (2010). Ethics in neuroscience training programs: views and models from Canada. Mind Brain Educ. 4, 20-27.

Samarasekera, I. V. (2009). Universities need a new social contract. Nature 462, 160-162.

Tairyan, K., Federico, C. A., Glover, G. H., Reiner, P. B., and Illes, J. (2010). No time to be ethical? Poster session to be presented at: Society for Neuroscience, Nov 13-17; San Diego, CA.

Tairyan, K., and Illes, J. (2009). Imaging genetics and the power of combined technologies: a perspective from neuroethics. Neuroscience 164, 7-15.

Zielinska, E. (2010). Are women better PIs? The Scientist 6, 71 .
Conflict of Interest Statement: The authors declare that the research was conducted in the absence of any commercial or financial relationships that could be construed as a potential conflict of interest.

Received:03 May 2010; paperpending published:04 May 2010; accepted: 29 July 2010; published online: 04 October 2010

Citation: Illes J, Tairyan K, Federico CA, Tabet $A$ and Glover GH (2010). Reducing barriers to ethics in neuroscience. Front. Hum. Neurosci. 4:167. doi:10.3389/ fnhum.2010.00167

Copyright (C) 2010 Illes, Tairyan, Federico, Tabet and Glover. This is an open-access article subject to an exclusive license agreement between the authors and the Frontiers Research Foundation, which permits unrestricted use, distribution, and reproduction in any medium, provided the original authors and source are credited. 